\title{
Situación de los egresados de la ENEO UNAM \\ en el mercado laboral 2003-2007
}

\section{ENEO-UNAM Graduates 2003-2007 labor market situation}

Mtra. Ma. Cristina Muggenburg Rodríguez V*. Mtra. Iñiga Pérez Cabrera** Lic. Esp. Pablo Ortiz Acevedo***

${ }^{\star}$ Profesor de carrera T.C.A. ENEO-UNAM, **Profesor de carrera T.C. Asociado C ENEO-UNAM,

***Especialista en Enfermería de Rehabilitación

\section{Resumen}

Introducción: El análisis del mercado laboral de los egresados de la ENEO UNAM, permite retroalimentar el diseño de los planes de estudio, en el presente se describen las tendencias de los indicadores laborales observados en los seguimientos de 2003 a 2007.

Metodología: Estudio descriptivo de cinco generaciones de egresados, con una muestra promedio anual de 66 casos, abordados a través de interrogatorio indirecto con un instrumento integrado con variables como: titulación, empleo, categorías e instituciones contratantes, salario, actividades desempeñadas y sustento de la práctica profesional; procesadas con el apoyo de estadística descriptiva.

Resultados: Las cifras anuales de empleo muestran valores cercanos al 89\%, con diversas categorías de contratación, pero con tendencia ascendente en el caso de la licenciatura, así como en el caso de los salarios superiores a los nueve mil pesos mensuales. Los principales contratantes son: Ia Secretaría de Salud y el Sector privado; las actividades más desempeñadas son los cuidados orientados a la resolución de necesidades y de alteraciones patológicas, así como al apoyo integral del paciente. Los egresados reconocen que los aprendizajes son el sustento prioritario de su práctica profesional.

Conclusiones: La situación laboral de los egresados de la ENEO es favorable al ser analizada en un contexto internacional incierto, ya que las tendencias observadas muestran cifras con valores superiores a los de otras profesiones.

Palabras clave: Mercado de trabajo, egresados de licenciatura en enfermería, desempeño laboral. 


\section{Abstract}

ENEO-UNAM Graduates 2003-2007 labor market situation Introduction: The analysis of the labor market for the ENEO-UNAM graduates allow us feedback our study plan designs. In this study, we describe some related trends between 2003 and 2007.

Methodology: This is a descriptive study involving five ENEO-UNAM graduates generations with a yearly sample average of 66 cases. We studied variables such as: graduating process; employment; hiring categories and institutions; salaries; activities performed; and professional practice support, all through an indirect interview questionnaire. We also obtained the corresponding descriptive statistics.

Results; Yearly employment figures show valves close to 89\% among various hiring categories, but with a tendency towards the bachelors level and salaries over $\$ 9.000 .00$ Mexican pesos per month. The principal employers were: The Health secretary and the private sector. The most frequently performed activities were needs resolution oriented care; pathological alterations; and patient integral support to their professional practice.

Conclusions: Considering the uncertainty of the international context, the labor situation of the ENEO-UNAM graduates is favorable since the observed tendencies show figures with better values in comparison to other professions.

Key words. Labor market, nursing bachelor graduates, Labor performance

\section{INTRODUCCIÓN}

El seguimiento de egresados de la ENEO entre otros aspectos se interesa por la situación de sus exalumnos en el mercado laboral, cumpliendo con el propósito de evaluación institucional externa, que le permite retroalimentar el proceso de formación; el análisis de las tendencias observadas podrá guiar el diseño de los planes de estudio sin soslayar los valores de la profesión y las necesidades sociales del país.

El mercado de trabajo es uno de los componentes de la economía y expresa las relaciones que se establecen entre los elementos que participan en la producción de bienes y servicios, como son los empleados y los empleadores; sin embargo el mercado de trabajo no se comporta de manera homogénea para todos los individuos, ya que personas con el mismo perfil profesional cumplen funciones muy disímiles en el mercado laboral ${ }^{1}$.

La teoría del capital humano percibe la acción escolar como un acto de inversión que debe ser rentable, este "capital" debe ser retribuido cuando el egresado forma parte del mercado laboral profesional; esta línea está más apegada a la perspectiva unidimensional de la Economía de la Educación que a una perspectiva humanista ${ }^{2}$, por lo que al analizar el comportamiento de los egresados como estrategia de retroalimentación de los planes de estudio, se puede reconocer la estrecha relación entre la formación profesional y las exigencias ocupacionales ${ }^{3}$, pero también se puede afirmar que no es determinante, los centros educativos se preocupan por formar alumnos competentes que puedan encontrar espacio en el mercado de trabajo pero también se interesan por el crecimiento y desarrollo profesional, en el caso de la Enfermería no solo se preparan alumnos con capacidades técnicas que respondan a las demandas de las instituciones de salud existentes, sino que también se busca la formación de egresados que valoren el ejercicio autónomo de su profesión, quienes trabajen en forma interdependiente con otros profesionales de la salud, con responsabilidades asignadas en el caso de tratamientos médicos y con responsabilidades propias en el caso de la oferta de cuidados de enfermería ${ }^{4}$.

La teoría del capital humano, ofrece bases para el análisis de las relaciones entre educación y trabajo, pero tiene algunas limitaciones que han sido abordadas por teorías emergentes, en las cuales se argumenta que aún cuando los individuos posean un gran capital humano las condiciones de trabajo no siempre responden a este ${ }^{5}$.

En el ámbito nacional y bajo el liderazgo de la ANUIES se han desarrollado diversos estudios de egresados que 
abordan tres aspectos: inserción de los egresados en el mercado de trabajo, desempeño profesional y opinión sobre la formación recibida ${ }^{6}$.

Otras instituciones, tanto de carácter político como educativo, nacionales e internacionales, también se han dado a la tarea del estudio de los mercados de trabajo profesionales o del seguimiento de sus egresados, siguiendo esquemas similares y/o complementarios, como es el caso de la UNAM ${ }^{7,8,9}$, la EAFIT de Medellín, Colombia $^{10}$, la CONICET de Argentina ${ }^{11}$, la Universidad Juárez Autónoma de Tabasco ${ }^{12}$, la OlT ${ }^{13}$ y el $\mathrm{ClE}^{14}$, entre otras.

En el campo de la Enfermería la UNAM ha llevado a cabo diversos estudios de egresados como en el caso de FES Zaragoza ${ }^{15}$ y de la ENEO ${ }^{16,17}$; además ha iniciado los estudios de empleadores de Enfermería ${ }^{18}$.

También se cuenta con un estudio sobre el desperdicio de capacidades y habilidades en licenciados en Enfermería, desarrollado a partir de bases datos de ANUIES y del XII Censo General de Población y Vivienda. ${ }^{19}$

En este contexto se revisa la situación de los egresados de la ENEO en el mercado laboral de 2003 a $2007^{20}$, identificando: condiciones del empleo, actividades realizadas y actitudes profesionales.

\section{METODOLOGÍA}

Estudio descriptivo en egresados de la licenciatura de la ENEO-UNAM de cinco generaciones encuestadas año con año desde 2003 hasta 2007, a los tres años de la terminación de sus estudios; con una población de 1035 egresados y una muestra aleatoria de tipo sistemático de 330 casos; los datos se recolectaron en el plantel escolar en la mayoría de los casos, previa invitación telefónica; se aplica un interrogatorio indirecto con un instrumento validado (validez de contenido y de criterio), con preguntas cerradas y tipo likert que exploran variables como: titulación, empleo, categoría de contratación, salario percibido, institución contratante, actividades realizadas con mayor frecuencia y sustento de su práctica; con el uso de escalas nominales y ordinales. El proceso de análisis de los datos se apoya en pruebas de estadística descriptiva. Investigación con riesgo mínimo.

\section{RESULTADOS}

Los seguimientos anuales de las diferentes generaciones de los egresados de la ENEO han permitido visualizar el desempeño profesional en el marco laboral y formativo, contando con informes disponibles en la biblioteca de la
ENEO desde 1994 hasta 2007, en los que se aprecian características personales, formación académica, condiciones laborales y desempeño profesional, pero el presente informe se concreta a la descripción y análisis de tendencias observadas en el mercado de trabajo que ocupan los egresados de la ENEO y se buscan explicaciones congruentes con experiencias nacionales e internacionales.

El porcentaje de empleo de los egresados por año aún cuando presenta variaciones que oscilan desde el $75 \%$ hasta el 95\%, tiende ligeramente al alza y se mantiene en valores cercanos al $89 \%$.

Las categorías de contratación van desde el nivel de auxiliar de enfermería hasta cargos administrativos, incluyendo otras categorías como: pasantes, enfermeras generales, licenciadas y especialistas, con tendencias ascendentes en el rubro de las licenciadas y las especialistas y tendencias descendentes en el nivel de auxiliares de enfermería (gráfica No.1)

En cuanto al salario mensual percibido se observa una tendencia marcada al alza en el caso de los montos que van de los \$ 9000.00 hasta los $\$ 16000.00$ y tendencia a la baja en el caso de los salarios inferiores a los $\$ 4000.00$ (gráfica No.2).

Las instituciones contratantes son del sector público y privado, en el primer caso predominantemente la Secretaría de Salud y el Instituto Mexicano de Seguridad Social; en el caso del sector privado, además de diversas instituciones asistenciales reconocidas, los egresados trabajan en forma independiente o a través de agencias particulares de atención domiciliaria (gráfica No.3).

El índice de titulación de los egresados muestra una tendencia positiva, que oscila desde $57 \%$ hasta $71 \%$, la obtención del título se alcanza prioritariamente entre el año y los dos y medio años del egreso, las opciones de titulación son varias, las más solicitadas son: Examen por áreas del conocimientos (EPAC), Proceso Atención de Enfermería (PAE) y Tesis, pero a partir del año de 2005 se aprueban nuevas opciones de titulación con el fin de favorecer el proceso oportuno de titulación ${ }^{21}$.

Respecto a las actividades que realizan con mayor frecuencia mencionan: cuidados de enfermería sencillos orientados a la resolución alteraciones en el enfermo, cuidados de enfermería complejos orientados a la resolución de alteraciones en el enfermo, cuidados de enfermería enfocados al apoyo integral del paciente y cuidados de enfermería sencillos enfocados a la resolución de necesidades fisiológicas de la persona. 
En cuanto a la congruencia identificada por los egresados entre la formación recibida y el mercado laboral, la mayoría consideró que es mucha o regular, ubicando el juicio de mayor congruencia en los seguimientos de 2005 y 2006, y de congruencia regular en 2003, 2004 y 2007, de hecho ambos valores se presentan compensados.

Por otra parte los egresados opinan que la calidad de la formación teórica es superior a la de la formación prác- tica, apreciación que se ha mantenido en los años analizados, aunque con una tendencia de ligera mejoría en la formación práctica (gráfica No.4).

En relación al sustento de su práctica profesional, los egresados manifiestan que su desempeño se apoya prioritariamente en sus aprendizajes, en las rutinas establecidas en los servicios o en el seguimiento de las órdenes médicas; las tendencias observadas en el periodo en

\section{Gráfica 1. Categorías de contratación por año.}

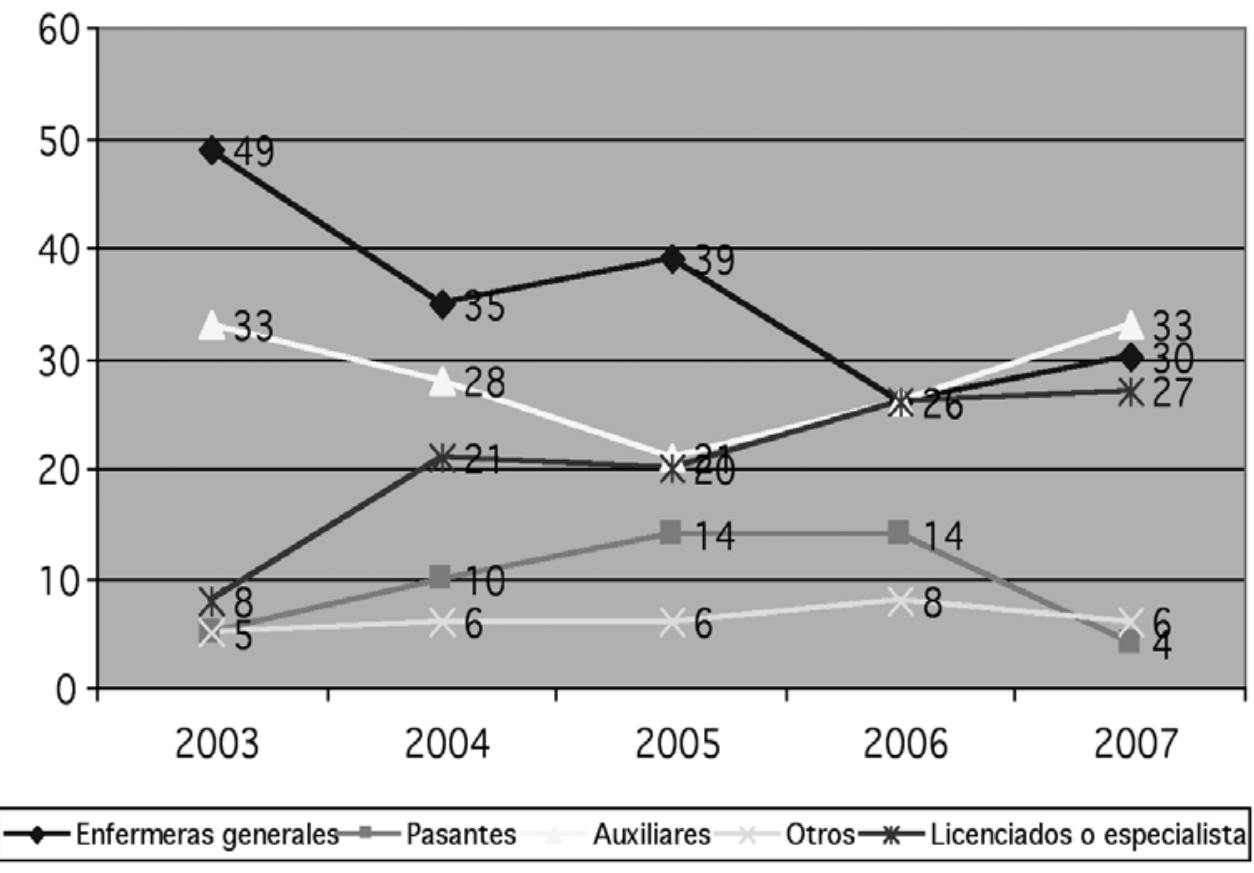

\section{Gráfica 2. Salarios mensuales por año.}

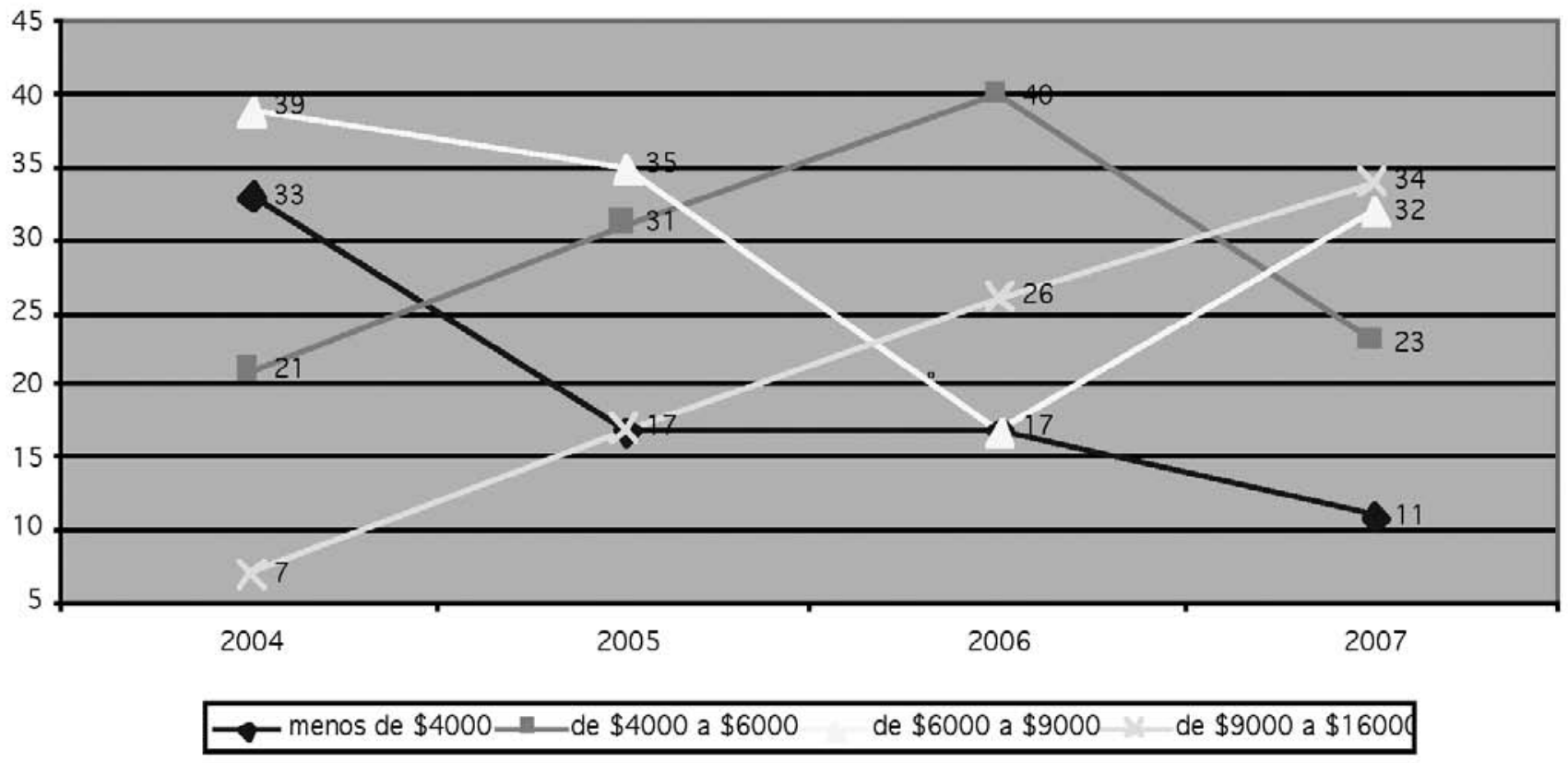


Gráfica 3. Principales instituciones contratantes.

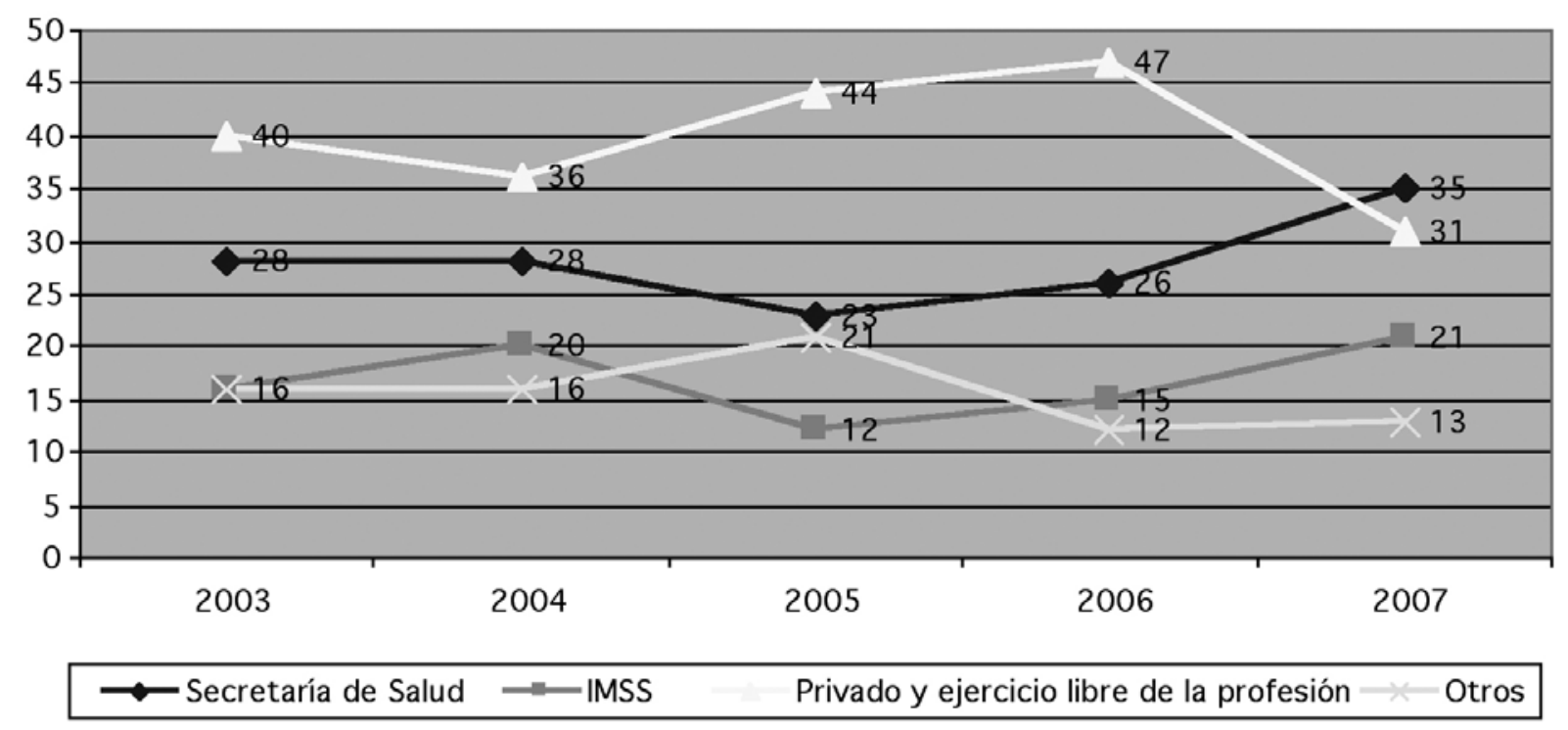

Gráfica 4. Calidad de la formación teórica y práctica.

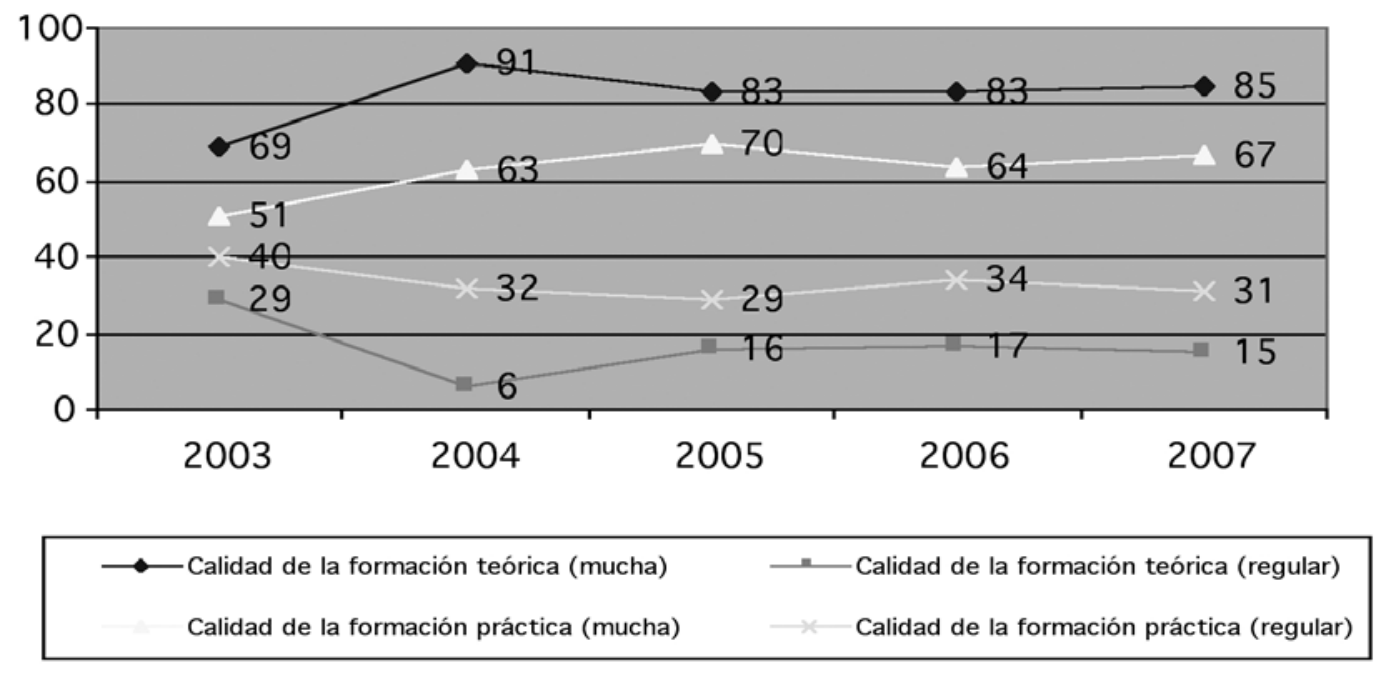

cuestión muestran que los aprendizajes son los elementos que mantienen la mayor frecuencia, en cuanto al seguimiento de rutinas establecidas en el servicio hay un ascenso al inicio del periodo analizado, pero pierde fuerza a partir del 2005; por otra parte, el seguimiento de las órdenes médicas ha estado presente pero con frecuencias inferiores a los otros dos parámetros analizados (gráfica No.5).

\section{DISCUSIÓN DE RESULTADOS.}

Los porcentajes observados en los egresados de la ENEO en cuanto al empleo aún muestran cifras y tendencias menos desfavorables que en otros profesionales recién egresados 22 23, situación que coincide con la información referida por el Consejo Internacional de Enfermería (CIE), en la que se señala que en los Estados Unidos y en la Comunidad Europea hay tendencia al déficit de enferme- 


\section{Gráfica 5. Sustento para la actuación en la práctica profesional de los egresados por año}

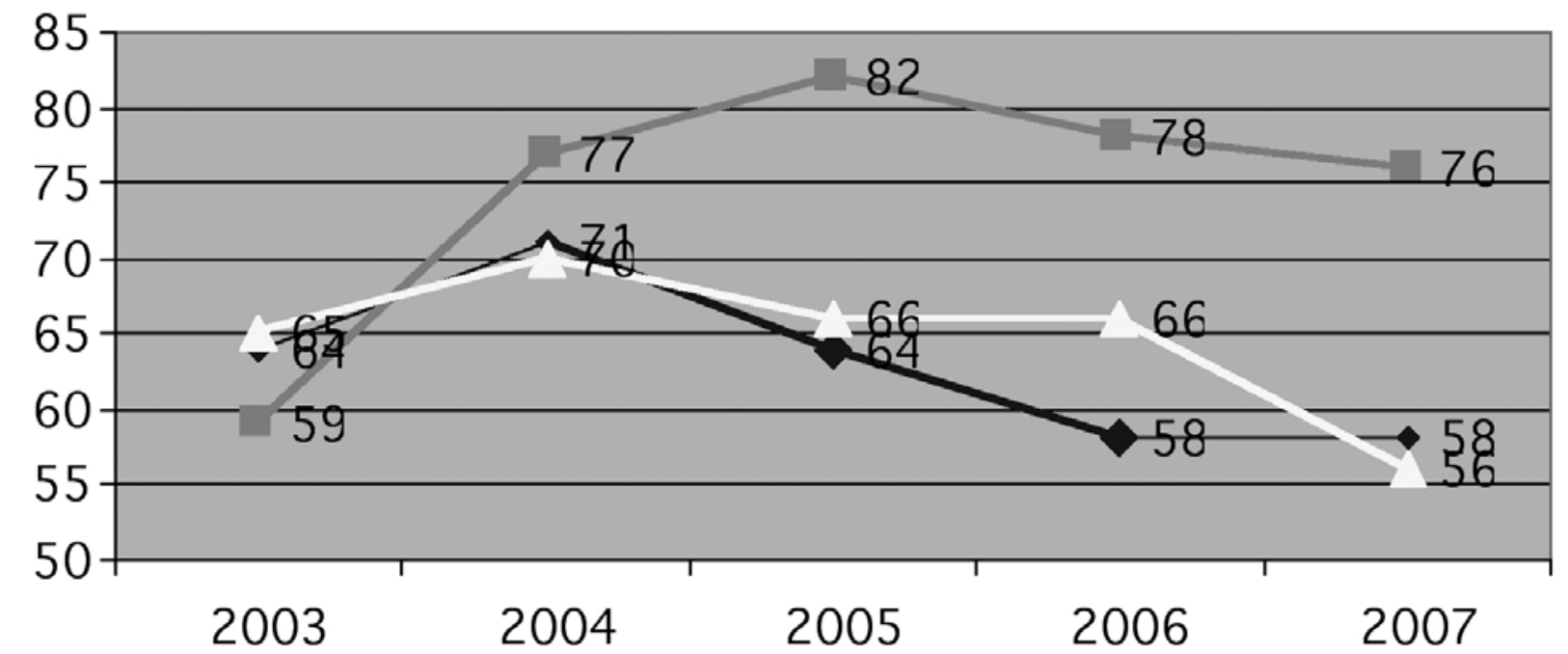

$\longrightarrow$ Rutinas establecidas en el servicio Órdenes médicas

ras y puestos vacantes ${ }^{24}$. Sin embargo el comportamiento actual de los egresados de la ENEO no debe verse exclusivamente con complacencia, sino que se debe ubicar en el marco internacional y en la grave situación económica que atraviesa este mundo globalizado.

Al analizar las categorías de contratación, en el caso de los egresados de la ENEO, se encuentra con ascenso en las plazas de licenciatura y descenso en las contrataciones como auxiliar de enfermería, situación que puede estar favorecida por lo índices de titulación, que tienden a alcanzar cifras cercanas al 70\% y por la creación reciente de políticas para el reconocimiento profesional de Enfermería por el sector gubernamental, mismas que tenían un gran rezago. ${ }^{25}$ Sin embargo la tendencia favorable para las contrataciones de carácter profesional no debe soslayar el fenómeno de subempleo que también está presente en algunos egresados, quiénes por asignación institucional desempeñan funciones de menor complejidad con subutilización de las capacidades adquiridas en el proceso de formación educativa, fenómeno que ha sido estudiado por Nigenda. ${ }^{26}$
La presencia de subempleo es un problema con riesgo de incremento como consecuencia de las medidas de contención de costos aplicadas por los empleadores, tanto en el sector público como privado. ${ }^{27}$ El ejercicio libre de la profesión es practicado por los egresados con mayor frecuencia que en años anteriores, pero esta forma de empleo los deje en desventaja en muchas ocasiones por no contar con mecanismos de seguridad social y con estructuras administrativas y legales que los protejan.

La tendencia positiva de ingreso mensual, paralela a la asignación de mejores plazas, es apreciada por la recuperación económica y por el reconocimiento profesional que conlleva en una profesión que día con día lucha por el lugar que merece en función de la responsabilidad ejercida en el cuidado para la salud de las personas. Sin embargo, el incremento observado no debe propiciar una actitud conformista, sino abierta al progreso económico, sin olvidar que la capacitación continua y el ejercicio calificado de la profesión son elementos básicos para demandar un incremento salarial acorde al ejercicio profesional desempeñado. 
El crecimiento de las contrataciones por el sector privado ya ha sido señalado en forma reiterativa y es una realidad inminente derivada de políticas internacionales con algunas consecuencias que deben ser reguladas por el sector salud y previstas por el sector educativo ${ }^{28}$.

La relación observada entre titulación y contratación y titulación y mejores oportunidades laborales señalada por otros autores ${ }^{29}$, también ha sido constatada en los estudios de Seguimiento de egresados de la ENEO.

Las actividades desempeñadas con mayor frecuencia, orientadas a actividades que buscan solucionar déficit en el individuo y ofrecer apoyo integral cumplen con el perfil de formación profesional esperado ${ }^{30}$, sin embargo la baja realización de actividades educativas orientadas a la prevención marcan una señal de alerta, por la necesidad del desempeño de estas actividades en congruencia con políticas nacionales e internacionales ${ }^{31}$.

A pesar de los cambios acelerados, derivados del desarrollo tecnológico, que se observan en los diversos escenarios de trabajo ${ }^{32}$, los egresados de la ENEO manifiestan congruencia entre su formación y el mercado laboral, pero el reconocimiento de la calidad de la formación teórica sobre la práctica, puede estar relacionado con variantes, que responden al desarrollo tecnológico de los campos de trabajo, lo que sugiere que los centros educativos propicien los aprendizajes reflexivos de los aspectos básicos del ámbito profesional ya que los aprendizajes específicos quedan rebasados en forma inmediata por el acelerado desarrollo tecnológico.

El reconocimiento de los aprendizajes como sustento predominante de la práctica profesional resulta halagador para los centros formadores y para el crecimiento de la profesión de Enfermería, misma que además del trabajo interdisciplinario y multidisciplinario, desarrolla trabajo independiente orientado al cuidado para la salud, eje de su identidad profesional.

\section{CONCLUSIONES}

La situación laboral de los egresados de la Escuela Nacional de enfermería de la UNAM es favorable al ser analizada en un contexto internacional incierto, ya que las tendencias observadas en el comportamiento del empleo muestran cifras con valores superiores al de otras profesiones, sin embargo la posición de alerta para los centros formadores, instancias reguladoras del sector salud y para las mismas enfermeras debe mantenerse por la situación que se prevé en lo general para el empleo y la economía en esta nueva etapa de un mundo global en crisis.

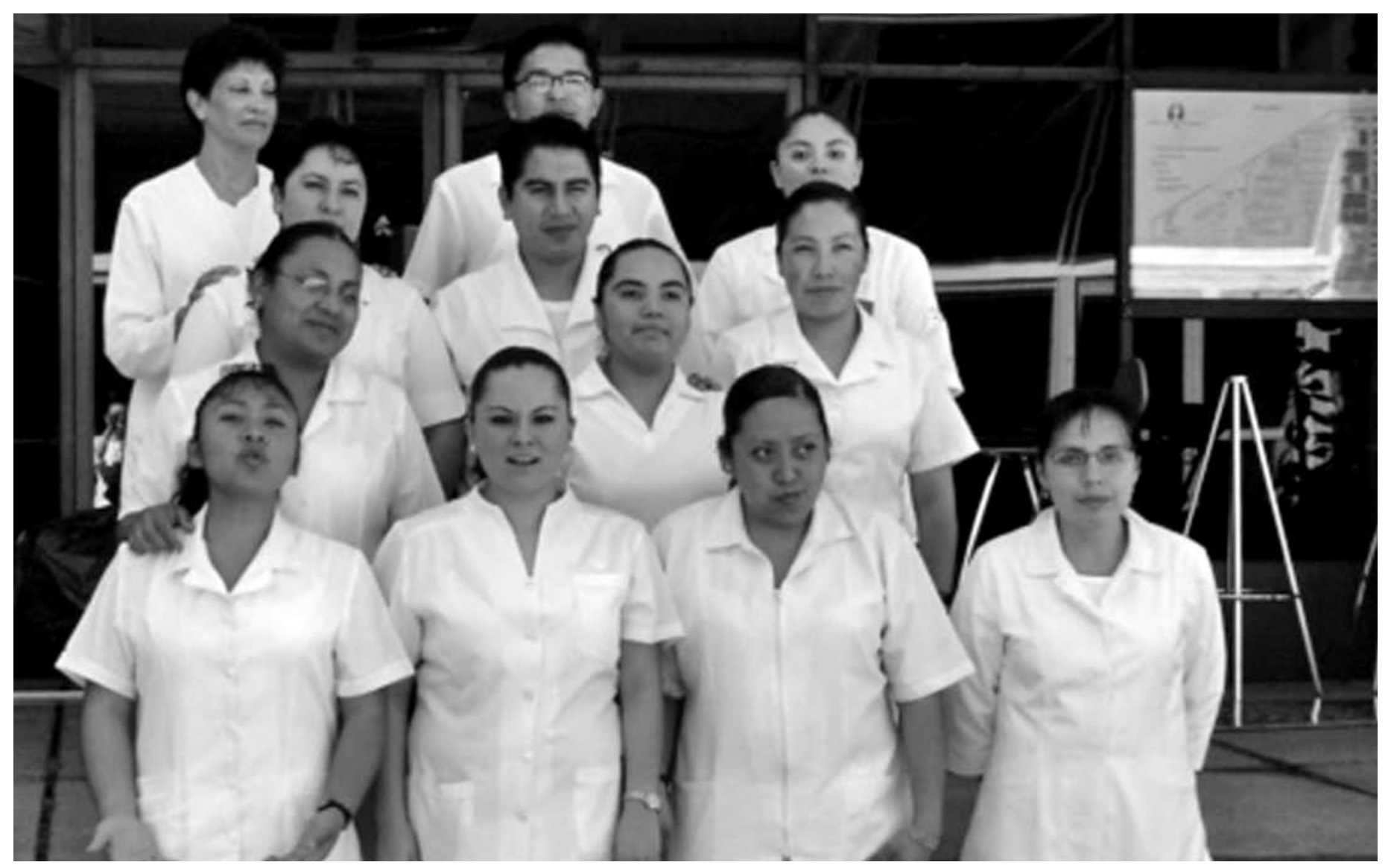




\section{REFERENCIAS BIBLIOGRÁFICAS}

1 Gómez-Ocampo, M. Educación superior, mercado de trabajo y práctica profesional. Revista de Educación Superior 45 ANUIES, enero- marzo 1983

2 Díaz-Barriga, A. Empleadores de Universitarios. Un estudio de sus opiniones. México. Centro de Estudios sobre la Universidad UNAM-Porrúa. 2000 p.52-55

3 Ibidem. p.60-63

4 Colliére, M.F. Promover la vida. Madrid. McGraw-Hill/ Interamericana de España 1993 p.144-156.

5 Navarro Leal, M.A. Consideraciones teóricas para el estudio de egresados. En: ANUIES. Esquema Básico para estudios de egresados. Biblioteca de la Educación Superior. México: 1998 p.11-18

6 Valente-Nigrini, Giovana y Varela-Petito, Gonzalo. Diagnóstico sobre el estado actual de los estudios de egresados. México, ANUIES: Noviembre 2003 p.7-8.

7 Díaz Barriga, Ángel. Empleadores de Universitarios, un estudio de opiniones. México, CESU-Miguel Ángel Porrúa: 1995 (Reimpresión 2000). pp.138

8 Valle Flores, Ángeles (coordinadora). Las profesiones en México frente al mercado de trabajo. Los retos de la formación. México, UNAM-CESU: 2004. pp.181

9 Pacheco Méndez Teresa y Díaz Barriga Ángel. La profesión universitaria en el contexto de la modernización. México, Pomares:2005 pp.159

10 Lopera, Carlos Mario. Los estudios sobre seguimiento de egresados en los procesos de mejora continua de la actividad universitaria. Monterrey, México (16 de marzo de 2005) p.9-10 http://www. gradua2.org.mx/docs/monterrey/Ponencia_U_Catolica_de_Col_GRADUA2_Mexico.doc

11 lbídem. p.17

12 Fortalecimiento de la Vinculación con Egresados y Organismos Empleadores. www.dacs.ujat.mx/informe_actividades/informe_06_07/mejoramientoV.pdf.

13 OIT. Extracto del comunicado de prensa de la OIT titulado: Un nuevo análisis de la OIT detecta incertidumbre creciente y cambio acelerado en el mundo de trabajo. Junio de 2006. www.lo.org

14 Consejo Internacional de Enfermería (CIE) Problemas mundiales de oferta y demanda de enfermeras. Socio Economic News. Sew News Boletines recientes del CIE. Arch. del Sew News. Sew: Vol.1 enero-marzo 2003

15 González Velázquez, María Susana y cols. Seguimiento de Egresados de la Licenciatura de Enfermería. Desarrollo Científico en Enfermería Vol. 17 No.3Abril: 2009 p.106109
15 Müggenburg, Cristina. Pérez Cabrera, Iñiga y cols. Seguimiento de egresados de la licenciatura de la ENEOUNAM formación y desempeño laboral (2003-2006). Revista Enfermería Universitaria ENEO-UNAM Vol.5, Año 5, 2008 p.21-29

17 Informes: Seguimientos de Egresados de la Licenciatura de la ENEO-UNAM de 2003 a 2007. Biblioteca de la ENEO UNAM

18 Crespo Knopfler, Silvia y cols. Percepción de empleadores sobre el Licenciado en Enfermería de la Facultad de Estudios Superiores Zaragoza. Revista Enfermería Universitaria ENEO-UNAM Vol. 6, Año 6, No. 1, 2009 p.7-11

19 Nigenda, Gustavo y cols. Enfermeras con licenciatura en México: estimación de los niveles de deserción escolar y desperdicio laboral. Salud Pública Méx. Vol.48 (1) 2006 p.22-29

20 Informes: Seguimientos de Egresados de la Licenciatura Op.cit.

21 Rubio Domínguez, Severino. Primer Informe de actividades de la ENEO UNAM 2007. p.49

22 Bouzas Ortiz, Alfonso. La crisis hace peligrar un millón de empleos. Gaceta UNAM, 10 de noviembre de 2008 p.8

23 Lozano Alarcón, Javier. En: Velasco Elizabeth; Iniciativa privada y sindicatos por empleo y paz laboral. La Jornada, 13 de noviembre de 2008

24 Consejo Internacional de Enfermería (CIE) Op.cit.

25 Diario Oficial de la Federación. México. 22 de diciembre de 2004

26 Nigenda Op.cit.

27 Consejo Internacional de Enfermería (CIE) Op.cit.

28 Informes: Seguimientos de Egresados de la Licenciatura Op.cit.

29 Díaz Barriga, A. Op.cit. p. 86

30 Plan de Estudios de la Licenciatura en Enfermería y Obstetricia ENEO UNAM 2003

31 Secretaria de Salud. Programa Nacional de Salud 20072012 (estrategia 2)

32 Valle Flores, A. Op.cit. p.51

\section{DIRECCIÓN PARA CORRESPONDENCIA}

Ma Cristina Muggenburg Rodríguez:

cmuggenb@yahoo.com.mx 


\title{
Apoyo emocional: conceptualización y actuación de enfermería, una mirada desde la perspectiva del alumno
}

\author{
Emotional support: conceptualization and performance \\ of nursing, a look from the perspective of the learner
}

Mtra. María Dolores Martínez Garduño*, Lic. Vicenta Gómez Martínez*, Lic. Piedad Siles Juárez*

*Profesora de tiempo completo Fac. de Enf. UAEMéx, ${ }^{* \star}$ Lic. en Enfermería, adscrita Centro Médico

Lic. Adolfo López Mateos, Instituto de Salud del Estado de México.

\section{Resumen}

Durante la formación de enfermería se hace hincapié en el apoyo emocional al paciente, destacando su importancia en el cuidado integral, sin embargo se carece de definición clara sobre este concepto y en muchas ocasiones, éste no se brinda. El objetivo fue identificar la conceptualización y cuidado de apoyo emocional que brindan los alumnos de la licenciatura en Enfermería.

Metodologia: El estudio fue descriptivo y transversal. El universo 210 alumnos. Las categorías del instrumento: conocimiento, identificación, planeación y evaluación de intervenciones para brindar apoyo emocional.

Una minoría define apoyo emocional como "Dar confianza, empatía y dar a conocer lo que le va a realizar al paciente".

Resultados: El conocimiento de la patología es prioritario (16\%) las acciones que realizan están encaminadas a favorecer la conversación como medio de disminución de la respuesta emocional (44\%); considera que es necesario ayudar o animar al paciente a expresar sus sentimientos (39\%); considera que se debe platicar con el paciente sobre algo ajeno a su enfermedad para brindar apoyo emocional 16\%; se debe brindar apoyo emocional realizando los procedimientos sin hablarle al paciente es la opinión del .55\%.

Es imprescindible hacer evidente la necesidad de crear una escala que facilite la valoración, y la evaluación de las intervenciones de enfermería con relación a las emociones sentidas y expresadas por los pacientes.

Palabras clave: enfermería, apoyo emocional, alumno. 